\title{
Screening and optimization of submerged fermentation of aspergillus species for kojic acid production
}

\section{Rehana Badar}

Institute of Molecular Biology and Biotechnology, The University of Lahore, Lahore

\section{Samiya Yaqoob}

Institute of Molecular Biology and Biotechnology, The University of Lahore, Lahore

Asma Ahmed ( $\sim$ asma.ahmed@imbb.uol.edu.pk)

The University of Lahore https://orcid.org/0000-0002-8432-746X

\section{Qurat UI Aan Shaoor}

University of Central Punjab, Lahore

\section{Research}

Keywords: Kojic acid, A. flavus and A. oryzae, pH, Quantitative, TLC, FTIR, HPLC

Posted Date: April 2nd, 2021

DOl: https://doi.org/10.21203/rs.3.rs-334019/v1

License: (a) (1) This work is licensed under a Creative Commons Attribution 4.0 International License.

Read Full License 


\section{Abstract}

Kojic acid is organic acid obtained from numerous species of Aspergillus through fermentation. This is among most demanding substances in cosmetic industries as an alternate to carcinogenic Hydroquinone and has grabbed a vital position in Pharmaceuticals, Food and Agriculture industries. Current experimental approach was designed for production and purification of Kojic acid crystals from $A$. flavusand A.oryzae and measured the effects of $\mathrm{pH}$, temperature, static and non-static (shaker) condition on Kojic acid yield in submergedfermentation. Significant yield of Kojic acid crystal was obtained by $A$. flavusas compared to A. oryzae. Optimized conditions were pH 4.5 for (A. flavus) and 3.5 for (A. oryzae) at $30{ }^{\circ} \mathrm{C}$ with 20 days of incubation. High yield of Kojic acid crystals were produced under static condition ( $16 \mathrm{~g} / \mathrm{L}$ in $A$. flavusand $11 \mathrm{~g} / \mathrm{L}$ in A.oryzae) in contrast to non- static (shaker) conditions $(6 \mathrm{~g} / \mathrm{L}$ in $A$. flavusand $5 \mathrm{~g} / \mathrm{L}$ in A. oryzae). Quantitative estimation of Kojic acid was done through Bentley's colorimetric method followed by TLC, FTIR and HPLC. This analysis was found successful after achieving the high yield of Kojic acid under optimized conditions.

\section{Introduction}

Kojic acid (5-hydroxy-2-hydroxymethylgamma-pyrone; KA) is considered as amajorsecondarymetabolitewhich is produced by the carbohydrates through various microorganisms, such as A.oryzae, A.flavus, and A. tamarii, as well as Penicilliumspecies andcertain bacteria (Hazzaaet al. 2013; Antonius et al. 2019).Kojic acid is an organic acid which covers almost all the fields, act as antibacterial, antifungal, anti-melanosis, and a chelating agent. It can be applied in various areas of science such as chemistry, health, food, and cosmetics; however, its current, reported uses are limited at commercial scale, as its despites at the market level were being developed about 40 years ago. However it is also marked as an organic chelation Agent used as a decolorizing agent. It is wellknown for its uses in Cosmetics Industry. Kojic acid is reported to have an antioxidant potential due to which is marked as to be used in the cosmetics industry, which has been further described as an alternative to hydroquinone for the lightening of the skin (Kady et al. 2014). This acid was basically discovered from the mycelia of $A$. oryzae which was grown on steamed rice (the term koji means steamed rice in Japanese) in the year 1907 (Burnett et al. 2010). Koji stands for rice in Japanese language and likewise the name of Kojic acid was given to this acid (Brtkoet al. 2004).

Kojic acid can be produced in large amounts by using various carbon and nitrogen base sources, based on agriculture waste under aerobic fermentation process. Till now among others glucose has been marked as the one of the best source as a raw material in order to attain higher yields of Kojic acid.Therefore use of some mutated strains of A.flavushad shown remarkable peaks $\mathrm{f}$ Kojic acid used potato,sago and corn starch etc. (Chaudharyet al.2014). Kojic acid has many economic applications in different eras, such as in the field of medical, it has been reported as having anti-bacterial and anti-fungal potentials. While in case of chemical industries it has been also used auspiciouslyfor the synthesis of azo-dyes andbiodegradable compounds. Furthermore in the field of foodindustries, it has been used as 
an anti-speckand anti- melanosis (blacking of product) agent for agricultural products (Hassan et al. 2014).

On commercial scale, Kojic acid synthesis was initiated by Charless Pfizer and Company of USA in 1955 which was the first company for this production. This finding was useful for further enhancement at commercial value, applications and production (Brtkoet al 2004; Bentley 2006). Recently the production of KA is marked by two Chinese and three companies in Japan, Switzerland, and the USA. Rapid growth of industries and discovery of the potential applications of KA along with its derivatives, generated a demands for the manufacture of this product KA greatly (Saeediet al. 2019).

According to global marketing report the use and production of cosmetic is increasing day by day. KA is used as primary constituent in skin products as it obstruct the synthesis of pigment, responsible for skin blackening (Masse et al. 2001). It is a good for the prevention of melanin production which ultimately safe man from freckles. However it was banned in Asia, later on due to carcinogenicity in by Drug Authority (Emamiet al, 2007).

Kojic acid is present in colorless prismatic needle form that can sublime in vacuum with no alteration in morphology. It has melting point ranging from $151^{\circ} \mathrm{C}$ to $154^{\circ} \mathrm{C}$, while the boiling point of this substance is $401.67^{\circ} \mathrm{C}$ at $760 \mathrm{mmHg}$. Furthermore the extensive use of this acid in the production of cosmetic are due to the ability of acting as a UV protector, which extensively work as a suppressor of hyperpigmentation in human and could have great potential to restrainer the formation of melanin, which is basically associated with its tyrosinase inhibition. This could also be developed as a chemo sensitizer in order to increase the potential of antifungal drugs or fungicides at market levels (Saeediet al. 2019).

According to Cryoscopy Method, molecular weight of Kojic acid is 142.1 and its maximum peak of UV Absorption Spectra is at 260-284 nanometer. KA is marked assoluble in Water, Acetone, Ethyl Acetate, and Ethyl Ether \& Ethanol and also slightly soluble in Ether, Alcohol-Ether mixture, Chloroform \& Pyridine etc. The solubility of this acid in Pyrimidine is very low, however, it is completely insoluble in Benzene (Neil, 2006).

Kojic acid has properties of weak acids and it is categorized as a multifunctional, reactive GammaPyrone. Its reactivity predominantly could be observed at all positions on a ring and a number of valuable industrial products can be made from it such as metal chelates, Ether, Pyrimidine, Pyridones, Azodies, Mannich Base etc. This is also reported as a strong antibacterial component, and had shown very strong control against gram positive bacteria (Zohriet al. 2018). Since beginning, several numbers of its chemical reactions have been studied for research purpose and its deliberate use for the benefit of humankind. It has ability to form salt with rare metal such as sodium, zinc, cooper, calcium, nickel and cadmium as its Hydroxyl group acts as weak acid at carbon 5 position. Carbon 5 side chain acts as primary alcohol whose reactivity is increased by neighboring oxygen atom in the nucleus (Chaudharyet al. 2014). 
It has strong reactions with amino acids to form conjugates, which exhibit a higher Tyrosine inhibition activity and stability as compared to KA alone, its derivatives are also reactive. (Mohammad et al. 2010; Noh et al. 2009). These derivatives are efficiently utilized in human medicines, veterinary medicines and synthesis of new and high biologically active compounds with preferable properties (Brtko, 2004). Secondary metabolites are the organic compounds which are not directly involved in the procedure of normal growth, progression and reproduction of an organism. Primary metabolites are responsible for the production of Secondary metabolite. Secondary metabolites play vital role in an organism's growth, reproduction as well as defense mechanism of living organism.

Plants use secondary metabolites in their defense system against herbs and humans utilize secondary metabolite as medicine, recreational drugs, texture preservative, food thickening and flavoring agent. (Pichersky and Gang, 2000; Göket al. 2013). Natural selection has been very important in the discovery of vital compounds which can give rise to new products. Organisms that inhabit vital biotopes produce natural products that perform specific functions and they can be used for vital secondary metabolite. (Schulz et al. 2002). However certain plants of Solonaceachad been reported to be used in drugs to cure diahorea and vomiting, phytoestrogens is secondary metabolite found in seed of Neem plant and act against insects (Chizzali and Beerhues 2012).

Aspergillus is one of the oldest known fungus known for the production of metabolites, acids and enzymes. Industrial utilization of microbial enzymes started 100 years ago in western world with the synthesis of Alpha Amylase by A. oryzae. These enzymes are used for numerous applications like cheese production, starch production, clarification of juice, food preservation and instant tea making. Thought the strains of oryzaehad been also reported for the production of lipase (Ahmed et al. 2019).Aspergillus is widely used for the production of organic acids such as citric acid, gluconic acid, KA at industrial level. These organic acids are used in food, soap, cream and in pharmaceutical as well. In course of survey different fungal species were used for its synthesis but Aspergillus species show fairly high yield of KA. They give deep red color with ferric chloride and upon continuous extraction produce crystals of KA (Brian, 1951).

According to 2014 Global marketing report, cosmetics business was 460 billion US dollar in 2014 and it is expected to reach to 675 billion US dollar in 2020. Hydroquinone used in skin care product has been replaced by Kojic acid (Emamiet al., 2007). Currently, Kojic acid is frequently used as a basic ingredient in skin lightening products because of its ability to block the formation of melanin, a pigment responsible for dark skin. (Masse et al. 2001).

The main objective of this experiment was to investigate the highest potential strains for the production of KA among A.flavusand $A$. oryzaeisolates through submergefermentations techniques. For that fermentation procedure was optimized by varying condition as static and non-static, temperature and $\mathrm{pH}$ of the media was also investigated.

\section{Results And Discussion}




\subsection{Effect of pH on A.flavus.}

In the past, a number of research analysis on synthesis of Kojic acid lead to the conclusion that numerous Aspergillus species have ability to produce Kojic acid. During this work the impacts of varying $\mathrm{pH}$ was closely examined on the production of Kojic acid in submerge fermentation. It was found that at $20^{\text {th }}$ day the maximum yield of Kojic acid crystals as $\mathrm{g} / \mathrm{L}$ was obtained $(13,16,9,7,5$ and 4 under static culture condition and $8,10,6,4$ and 3 ) under non-static at pH 3.5, 4.5, 5.5, 6.5, 7.5 and 8.5 correspondingly. Moreover maximum amount of Kojic acid crystals formulated from A. flavuswas obtained at pH 4.5 under Static and Non-static culture media. The highest yield of Kojic acidcrystals at pH 4.5 under static and Non-static conditions was $16 \mathrm{~g} / \mathrm{L}$ and $10 \mathrm{~g} / \mathrm{Lrespectively.} \mathrm{Increase} \mathrm{in} \mathrm{pH} \mathrm{of} \mathrm{the}$ culture media resulted negative impacts on quantitative concentration of Kojic acid and pH 4.5 was found to be most appropriate for the highest yield of Kojic acid. On the other hand, culture media of $A$. flavusat Non-static conditions gave low yield as compared to the ones treated at Static Conditions (Figure $3)$.

The subject research study results were found to be parallel to the results ofHazzaet al., (2013). Effect of high $\mathrm{pH}$ was significant on fungus metabolism. The metabolism of fungus shunted to some other pathways instead of Kojic acid production, so yield of Kojic acid reduced very much with high pH (Rosfarizanet al.,2010). A. flavusshowed highest yield of Kojic acidcrystals at pH 4.0. With an increase in $\mathrm{pH}$ of the culture media the growth of fungus and yield of Kojic acid ceased (Kady et al. 2014).

\subsection{Effect of temperature on A.flavus}

Yield of Kojic acid crystalswas strangely different at different temperature(s) (Figure 4) after 5, 10, 15 and 20 days. The growth of fungus was good at $25^{\circ} \mathrm{C}$ to $30^{\circ} \mathrm{C}$ and extraordinarily high at $30^{\circ} \mathrm{C}$ and unusually low at $40^{\circ} \mathrm{C}$. At day 20 the obtained yield of Kojic acid crystals was $6,12,5$ and $3 \mathrm{~g} / \mathrm{L}$ at $25^{\circ} \mathrm{C}, 30^{\circ} \mathrm{C}, 35^{\circ} \mathrm{C}$ and $40^{\circ} \mathrm{C}$ respectively. Highest yield of Kojic acid was $12 \mathrm{~g} / \mathrm{L}$ at $30^{\circ} \mathrm{C}$ (Gqalenet al., 1997 and Ito, Y., 2001).

\subsection{Effect ofpH on A.oryzae}

Yield of Kojic acid crystals significantly affected when high pH and conditions applied. At day 20, concentration of Kojic acid was 6, 4, 3, 2, 1 and $0.9 \mathrm{~g} / \mathrm{Lat} \mathrm{pH}$ 3.5-8.5 respectively under Static conditions, whereas, under Non-static conditions the yield was 5, 3, 2, 1.6, 1.1 and $0.5 \mathrm{~g} / \mathrm{L}$ at pH 3.5, 4.5, 5.5, 6.5, 7.5 and 8.5 respectively (Figure 5).

At $20^{\text {th }}$ day, the best yield of crystals of Kojic acid has been obtained from A.oryzae at pH $3.5(6 \mathrm{~g} / \mathrm{L}$ under static and $5 \mathrm{~g} / \mathrm{L}$ under non-staticconditions). Moreover, Static condition has shown good results of yield as compared to that of Non-static. Acquired results are similar to the results of Hazza, (2013) and Hassan et al., (2014). Same results was explained by Kadyet al., (2014), A.oryzaeand its strains with an increase in $\mathrm{pH}$ give low yield of acid and growth of fungus. A.oryzaeshow high growth on low pH i.e. 2 while high Kojic acid production is obtained at pH 3.5. As explained by Basappaet al., (1970), yield of 
Kojic acid severely reduced with a small deviation in $\mathrm{pH}$ and A.oryzae showed highest yield at pH 3.0 and increased $\mathrm{pH}$ decreased both yield of acid and fungal growth.

\subsection{Effect of temperature on A. oryzae}

Temperature adversely affects the yield of Kojic acid as well growth of $A$. oryzae. High growth of fungus was seen when temperature is $25^{\circ} \mathrm{C}$ to $30^{\circ} \mathrm{C}$ (Figure 6). At $30{ }^{\circ} \mathrm{C}$ the yield of Kojic acid was amazingly high and at $40{ }^{\circ} \mathrm{C}$ it was extremely low. The yield obtained at day 20 was $2,4,1.0$ and $0.2 \mathrm{~g} / \mathrm{L}$ at $25{ }^{\circ} \mathrm{C}$, $30^{\circ} \mathrm{C}, 35^{\circ} \mathrm{C}$ and $40^{\circ} \mathrm{C}$ correspondingly. Highest obtained yield was $4 \mathrm{~g} / \mathrm{L}$ at $30^{\circ} \mathrm{C}$ and lowest as $0.2 \mathrm{~g} / \mathrm{L}$ at $40{ }^{\circ} \mathrm{C}$. A decrease in Kojic acid concentration with increase in temperature was observed, whereas, $28^{\circ} \mathrm{C}$ was found to be the best temperature by El-Aasar (2006) in A. oryzae. Same results are reported by Devi et al., (2014) and Rosfarizan et al., (2010) that an increase in temperature shows significant effect on yield of Kojic acid.

\subsection{FTIR analysis of Kojic acid:}

FTIR Spectrum analysis of the sample showed the presence of various active functional groups, which had been indicated by the peaks which presents the presence of Amines N-H stretch (at 3253.59 $\mathrm{cm}^{-1}$ and $3179.41 \mathrm{~cm}^{-1}$ ) of Crystals. Samples also showed the presence of alkenes at $1629.86 \mathrm{~cm}-1$ $($ Cyclic-C=C). Furthermore these samples showed the presence ofalcoholic compound (O-H stretch) at 3600 wavelength $\mathrm{cm}^{-1}$ (Figure 8).

\subsection{HPLC of Kojic acid:}

The best results obtained by HPLC of $A$. flavusand oryzae contained the compound with the same retention time as that of Kojic acid standard (Figure 8). All the experimental analysis(HPLC, TLC and FTIR) indicated that Kojic acid was produced by both fungus.

\section{Materials And Methods}

\section{Experimental Lay Out}

Experiment was arranged by providing varying $\mathrm{pH}$ and Temperature in Static and Non-static conditions to attain Optimized conditions of fermentation Procedures for the highest yield of Kojic acid.

\section{Collection and Maintainance of Fungus}

In order to carried out this experimental analysis two species of Aspergillus (A. flavusand A. oryzae) were purchased from First Culture Bank of Pakistan, University of the Punjab Lahore. For the enhancement of the growth of mother culture, Sub-cultured by using potato dextrose agar (PDA) as the best source for the production of Micro-Organisms. Formation of Potato Dextrose Agar (PDA) media $3.9 \mathrm{~g}$ of PDA was dissolved in $100 \mathrm{ml}$ of water in a $250 \mathrm{ml}$ of Flask and were placed on hot plate for mixing until thoroughly dissolved in water. Subsequently, the flask containing the solution were cotton plugged and wrapped by 
aluminum foil. The flask was autoclaved for $1 \mathrm{~h}$, then poured into tubes and placed for ten min. After solidification of PDA solution, a loop of mother culture was taken and streaked on the Slants. These Slants were covered tightly and placed into incubator for 7 days at $30^{\circ} \mathrm{C}$. The whole procedure of Slant formation was conducted in a Laminar Flow to avoid contamination hence enhancing the number or growth of mother culture (Syarifuddin and Saidi, 2013).

\section{Preparation of Culture Media}

One liter of culture media was prepared by using $100 \mathrm{~g}$ ofglucose (as a carbon source), 5 gyeast (as a nitrogen source), $1 \mathrm{~g} \mathrm{KH}_{2} \mathrm{PO}_{4}, 0.5 \mathrm{~g} \mathrm{MgSO}_{4}$ and $10 \mathrm{ml}$ methanol. Aforementioned media being the high productive media was utilized by all referred Scientists (El-Aziz, 2013). To avoid the production of Aflatoxins, Methanol was used in the media solution (Madihah et al. 1998).

\section{Maintenance of $\mathrm{pH}$ and Temperature}

$150 \mathrm{ml}$ of Culture media was poured in $250 \mathrm{ml}$ flask and twelve such numbers of flasks were prepared. $\mathrm{pH}$ of these flasks were adjusted to $3.5,4.5,5.5,6.5,7.5$ and 8.5 by using $\mathrm{H}_{2} \mathrm{SO}_{4}$ and $\mathrm{NaOH}$. 2 such flasks were prepared for each $\mathrm{pH}, 01$ for Static and the other for Non-static (shaking) procedure. All flasks were labeled with ( $\mathrm{pH}$ and target procedure)-wise and inoculated with $A$. flavusand $A$. oryzae separately. Then 06 flasks containing the solution ranging from 3.5 to $8.5 \mathrm{pH}$ were placed on shaker at $30^{\circ} \mathrm{C}$ and likewise 6 others in an incubator at $30{ }^{\circ} \mathrm{C}$ for 20 days.

$150 \mathrm{ml}$ of Culture media was poured in 8 prepared $250 \mathrm{ml}$ flasks varying temperatures under 2 different procedures i.e. Static and Non-static (shaking). All flasks were labeled with temperature of 25, 30, 35 and $40{ }^{\circ} \mathrm{C}$ and target procedure wise and inoculated with A. flavusand A. oryzae separately in static and nonstatic conditions for about 20 days.

\section{Wet and Dry Mycelia Weight}

The flasks were removed from shaker and incubators and Mycelia mass was separated from culture and weighed (Weighing balance Model No. JJ224BC). The same mycelial mass was placed in oven to dry for $24 \mathrm{~h}$ and weighed again.

\section{Analysis of Kojic acid (KA)}

Colorimetric method was used for the quantification of Kojic acid (Bentlay, 1957). $1 \mathrm{ml}$ of diluted sample was mixed into $1 \mathrm{ml} \mathrm{FeCl}_{3}$ solution ( $1 \mathrm{~g}$ of $\mathrm{FeCl}_{3}, 6 \mathrm{H}_{2} \mathrm{O}$ in $100 \mathrm{ml}$ of $0.1 \mathrm{~N} \mathrm{HCL}$ ) (Sikem, N. D., 2013). Sample gave a reddish purple color due to reaction between hydroxyl and phenolic group as KA makes a complex with $\mathrm{FeCl}_{3}$ resultantly giving this purple color. Absorbance of the sample was measured at 500 nm by using UV- VIS Dual Split-Beam Spectrophotometer (Model No. UV2400PC) and equivalence was checked by Kojic acid standard curve(Crueger, A., 2006 and Bentley, R., 2006). 
Comparison of Standard Curve of self-prepared KAand prepared from standard KAobtained from PCSIR, Lahore was drafted to evaluate the comparative yield.10 different dilute solutions of Standard Kojic acid were prepared and placed in Spectrophotometer (Model No. UV2400PC) at $500 \mathrm{~nm}$ to measure absorbance of dilute solutions and plotted a graph between the concentrations of multiple dilute solutions and absorbance (Figure 1). Later, need-based dilute solutions of self-prepared Kojic acid (culture media) were prepared and likewise placed in Spectrophotometer (Model No. UV2400PC) at 500 nmto measure absorbance of dilute solutions. Trend analysis w.r.t. absorbance of dilute solutions was noted after intervals of 5 days and obtained O.D. of Standard and self-prepared Kojic acid were compared to evaluate the comparative yield for both.

\section{Extraction and crystallization of Kojic acid}

Extraction of Kojic acid crystals was carried out by filtration of culture media by Watt man filter paper No. 1. The left mycelial mass was further used for wet and dry weight procedure as mentioned above. The left over solution was treated with ethyl Acetate and placed at $5^{\circ} \mathrm{C}$ in refrigerator (Model No. FYLC400/FYLC-600)for $24 \mathrm{~h}$. Crystals of KA were seen next day at the bottom of beaker, than filtration was carried out to attain them which were oven dried at $80{ }^{\circ} \mathrm{C}$ for 01 hour (Figure $2 \mathrm{~A}$ and B) (Chaves, 2012).

\section{Thin Layer Chromatography (TLC)}

It was carried out to authenticate the presence of Kojic acid in culture media. Three Dilute solutions; One for Standard Kojic acid and two for A. flavusand A. oryzae (self-prepared Kojic acids) were prepared (1 ml water should contained $1 \mathrm{mg}$ of Kojic acid)in each dilute solution to further perform TLC procedure by using TLC plates coated with silica gel (Kisel gel160F 254) and a line marked with A (standard Kojic acid), B (Kojic acid prepared with A. flavus) and C (Kojic acid prepared with A. oryzae) (Fig 3 A). All 03 solutions were dropped on the plate and placed in mixture of water, acetic acid, acetone and ethyl acetate (1:1:3:5). After 15 minutes the plate was taken out and placed until dried. A solution of $\mathrm{FeCl}_{3}\left(1 \mathrm{~g} \mathrm{FeCl}_{3}\right.$ with $850 \mu \mathrm{l}$ of $\mathrm{HCl}$ and $100 \mathrm{ml}$ of water) was sprayed on TLC plate to obtain observable spots of poured Kojic acid (Figure $2 \mathrm{C}$ ).

\section{Fourier Transform Infrared Spectroscopy (FTIR)}

To further authenticate the presence of Kojic acid in extracted crystals, Fourier Transform Infrared Spectroscopy (FTIR)at FTIR spectrometer (a Shimadzu Prestige 2, Apodization: Happ-Genzel spectrometer) had been performed by ACRC Department of PCSIR laboratories, Lahore, Punjab, Pakistan. Each spectrum was recorded from 4000 to $500 \mathrm{~cm}^{-1}$ and 64 scans were accumulated for each spectrum.

\section{High Performance Liquid Chromatography (HPLC)}

It has been done as described by the protocol of Asma et al. , (2018). Crystals of Kojic acid has been dissolved in $80 \mathrm{~mL} \mathrm{C}_{2} \mathrm{H}_{5} \mathrm{OH}(80 \%)$, for one minute, filtered and stored at minus $20^{\circ} \mathrm{C}$ till analysis. $5 \mathrm{~mL}$ aliquot of stored extract was dried under vacuum at room temperature and resuspended in one $\mathrm{mL}$ 
$\mathrm{C}_{2} \mathrm{H}_{5} \mathrm{OH}(80 \%)$ and filtered through 0.45 um nylon 66 filter paper and injected $10 \mu \mathrm{L}$ from this solution into the HPLC system. Perkin-Elmer model of HPLC with binary LC pump of 250, an LC 600 auto sampler, a UV/V with spectrometric UV detector (at 270 nm) of LC- 290, PV Nelson 900 series INTERFACE, HewlettHewlettPakkrad 3394 Integration and a Bondapak C-18 column $(250 \star 4.6 \mathrm{~mm})$. Mobile phase was $0.5 \%$ orthophosphoric acid in $\mathrm{CH} 3 \mathrm{OH} ; 1 \mathrm{~mL} /$ minute flow rate was set and Aglycone content was measured using Quercetin dihydrate (95\%) as standard. Concentration was measured by using following formula

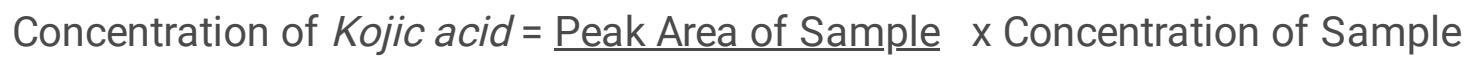

Peak Area of Standard

Patil et al. [13]. Twenty grams of onion sample, by removing dry leaves, was grounded with

\section{Declarations}

\section{Ethics approval and consent to participate}

Not applicable

\section{Consent for publication}

Not applicable

\section{Availability of data and materials}

- All data generated or analyzed during this study are included in this published article.

- Data sharing not applicable to this article as no datasets were generated or analyzed during the current study.

\section{Competing interests}

The authors declare that they have no competing interests

\section{Funding}

All authors funded equally in the design of the study and collection, analysis, and interpretation of data and in writing the manuscript.

\section{Authors' contributions}

$\mathrm{RB}=$ The acquisition, analysis and interpretation of data

SY= Conception or Design of the Work

$A A=$ The creation of new software used in the work; have substantively revised it 
QAS = Have drafted the work

\section{Acknowledgements}

Not applicable

\section{References}

Asma, A., Muhammad, G. and Noman, K. (2018).Isolation, Purification and Quantification of Quercetin and Primary Metabolites from Onion (Allium cepa L.). Proceedings of the Pakistan Academy of Sciences: Pakistan Academy of Sciences B. Life and Environmental Sciences 55 (1): 79-86.

Antonius R. B. Ola, GemaMetboki, Caterina S. Lay, YosephSugi, Philipi De Rozari,DodiDarmakusuma and EuisHolisotan Hakim. (2019). Single Production of Kojic acid by A. flavusand the Revision of Flufuran.Molecules2019, 24, 4200; doi:10.3390/molecules24224200.

Basappa, S. C., Sreenivasamurthy, V. andParpia, H. A. B. (1970).Aflatoxin and Kojic acid production by resting cells of $A$. flavusLink.Microbiology, 61(1), 81-86.

Bentley, R. (2006). From miso, sake and shoyu to cosmetics: a century of science for kojic acid. Natural product reports, 23(6), 1046-1062.

Brian, P. W. (1951). Antibiotics produced by fungi. The Botanical Review 17 (6): 357-430.

Burnett, C. L. (2010). Final report of the safety assessment of Kojic acid as used in cosmetics. International Journal of Toxicology. 29: 244S-273S.

Brtko, J., Rondahl, L., Fickova, M., Hudecova, D., Eybl, V., andUher, M. (2004).Kojic acid and its derivatives: history and present state of art. Central European Journal of Public Health, 12(SUPP), S16-S17.

Chaves, F. C., Gianfagna, T. J., Aneja, M., Posada, F., Peterson, S. W. and Vega, F. E. (2012).A. oryzae NRRL 35191 from coffee, a non-toxigenic endophyte with the ability to synthesize kojic acid. Mycological progress, 11(1), 263-267.

Chizzali, C. andBeerhues, L. (2012).Phytoalexins of the Pyrinae: Biphenyls and dibenzofurans.Beilstein journal of organic chemistry,8, 613-620. doi:10.3762/bjoc.8.68.

Chaudhary, J., Pathak, A.N. andLakhawat, S. 2014. Production technology and applications of Kojic acid.Annual Research and Review in Biology. 4(21): 31653196.

Crueger, W. and Crueger, A. (2006). Biotechnology: a textbook of industrial microbiology.

Devi, K. B. D., Vijayalakshmi, P., Kumar, B. V. andTalluri, V. P. (2014).Statistical Optimization of Kojic acid Production through Response Surface Methodology by $A$. flavususing Sago Starch Hydrolysate as a Carbon Source.Asian Journal of Applied Science And Engineering, 3(4), 421-428. 
El-Aasar, S. A. (2006). Cultural conditions studies on Kojic acid production by Aspergillusparasiticus. International Journal of Agriculture and Biology.8(4), 468-73.

El-Aziz, A. B. A. (2013).Improvement of Kojic acid production by a mutant strain of Aspergillusflavus. Journal of Natural sciences Research, 3(4), 31-41.

Kady, I. A., Zohri, A. N. A., andHamed, S. R. (2014).Kojic acid production from agro-industrial by-products using fungi. Biotechnology research international.2014. https://doi.org/10.1155/2014/642385.

Emami, S., Hosseinimehr, S. J., Taghdisi, S. M., andAkhlaghpoor, S. (2007). Kojic acid and its manganese and zinc complexes as potential radioprotective agents.Bioorganic and medicinal chemistry letters, 17(1), 45-48.

Gqaleni, N., Smith, J. E., Lacey, J., andGettinby, G. (1997). Effects of temperature, water activity, and incubation time on production of aflatoxins and cyclopiazonic acid by an isolate of $A$. flavusin surface agar culture. Applied and environmental microbiology, 63(3), 1048-1053.

Hassan, H. M., Saad, A. M., Hazzaa, M. M. and Ibrahim, E. I. (2014). Optimization Study for the Production of Kojic acid Crystals by A. oryzae var. effusus NRC 14 Isolate. International Journal of Current Microbiology and AppliedSciences, 3(10), 133-142.

Hazzaa, M. M., Saad, A. M., Hassan, H. M. and Ibrahim, E. (2013). High Production of Kojic acid crystals by isolated A. oryzae var. effuses NRC14. Journal of Applied Sciences Research, 9(3), 1714-1723.

Ito, Y., Peterson, S. W., Wicklow, D. T. andGoto, T. (2001).Aspergilluspseudotamarii, a new aflatoxin producing species in Aspergillus section Flavi.Mycological Research, 105(2), 233-239.

Masse, M. O., Duvallet, V., Borremans, M. andGoeyens, L. (2001).Identification and quantitative analysis of Kojic acid and arbutine in skin whitening cosmetics. International journal of cosmetic science, 23(4), 219-232.

Noh, J. M., Kwak, S. Y., Seo, H. S., Seo, J. H., Kim, B. G. and Lee, Y. S. (2009).Kojic acid-amino acid conjugates as tyrosinase inhibitors. Bioorganic \& medicinal chemistry letters, 19(19), 5586-5589.

Pichersky, E. and Gang, D. R. (2000). Genetics and biochemistry of secondary metabolites in plants: an evolutionary perspective. Trends in plant science, 5(10), 439445.

Rosfarizan, M., Ariff, A. B., Hassan, M. A. andKarim, M. I. (2010). Influence of pH on Kojic acid fermentation by Aspergillusflavus. Pakistan Journal of Biological Science, 3, 977-82.

Schulz, B., Boyle, C., Draeger, S., Römmert, A. K. and Krohn, K. (2002). Endophytic fungi: a source of novel biologically active secondary metabolites Paper presented at the British Mycological Society symposium on Fungal Bioactive .Compounds, held at the University of Wales Swansea on 22-27 April 2001. Mycological Research, 106(9), 996-1004. 
Figures

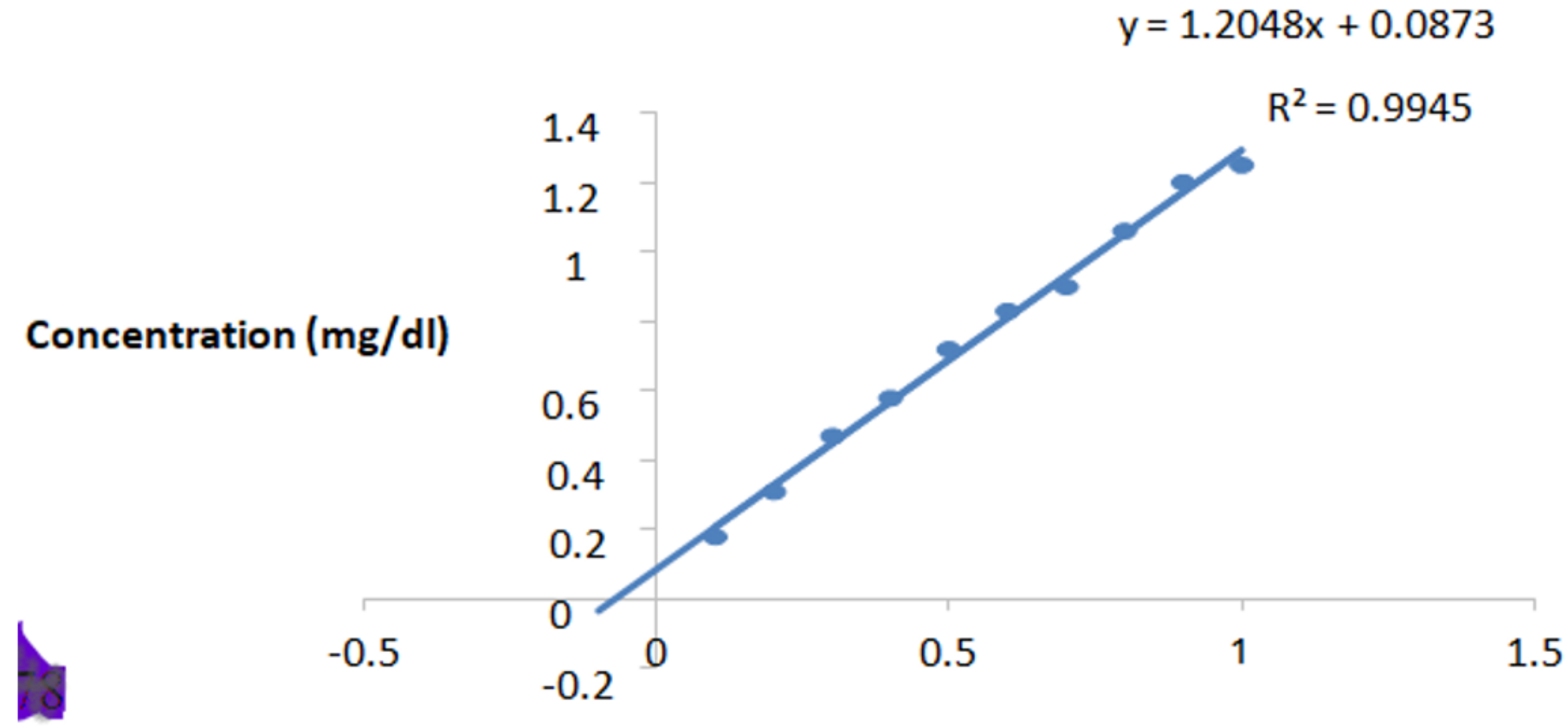

Absorbance

Figure 1

Standard Curve of Pure Kojic acid
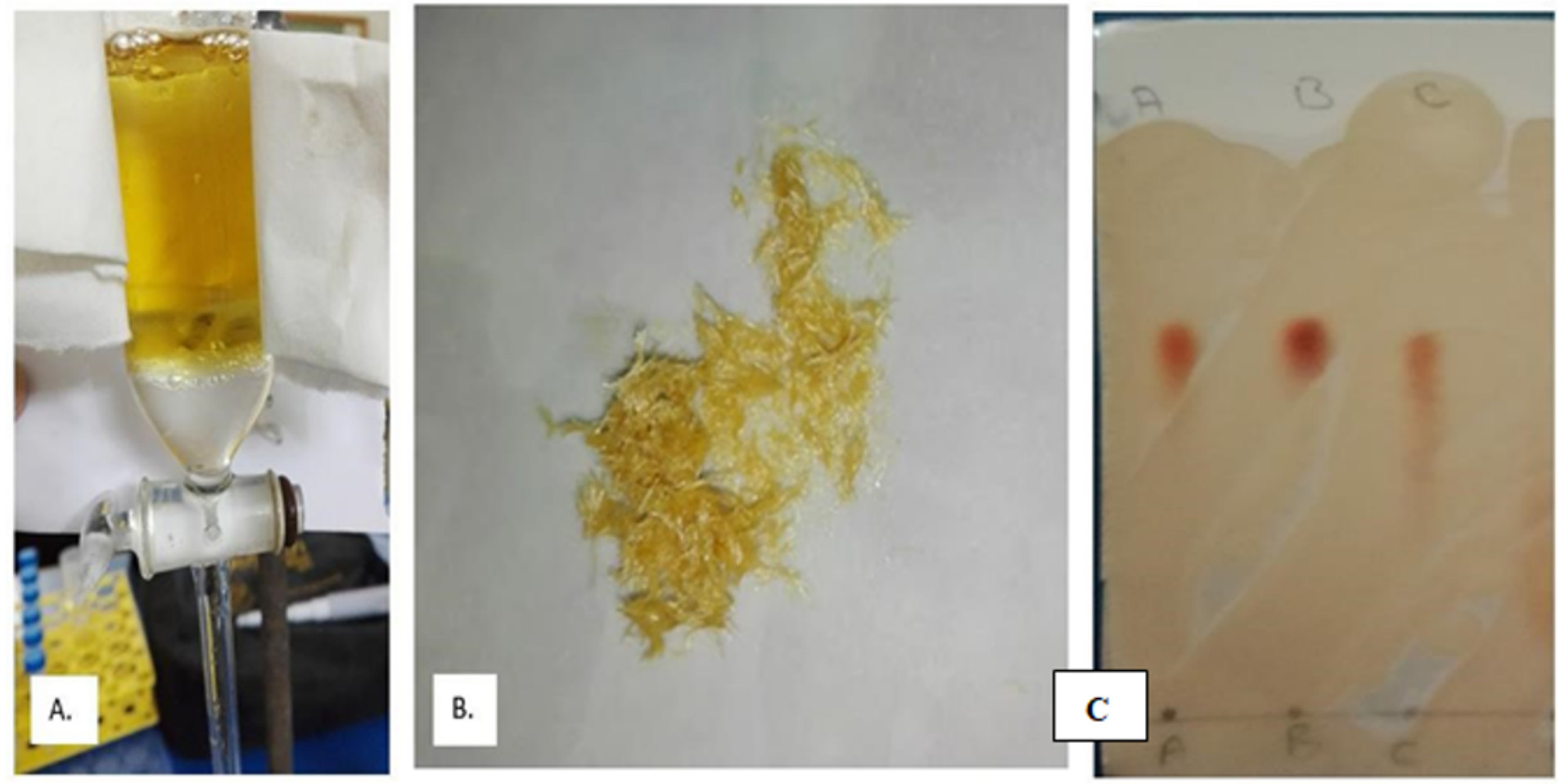

Figure 2 
A: extraction of Kojic acid; B:Kojic acid Crystals C: Kojic acid on TLC plate.

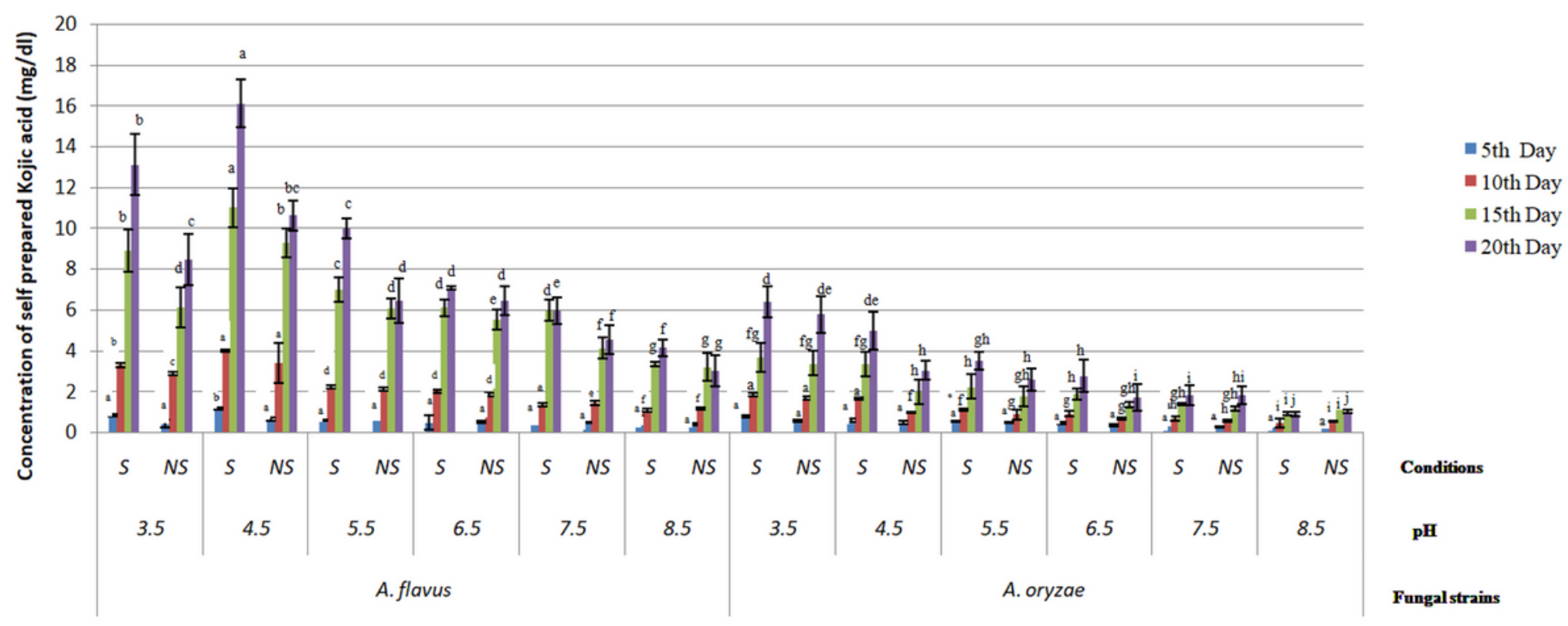

Figure 3

Concentration of self-prepared Kojic acid from A.flavus and A. oryzae in static and in non-static conditions on different days at different pH S= static, NS= non-static, a= Maximum amount of Kojic acid (as per statistical analysis), $\mathrm{j}=$ Least amount of Kojic acid (as per statistical analysis)

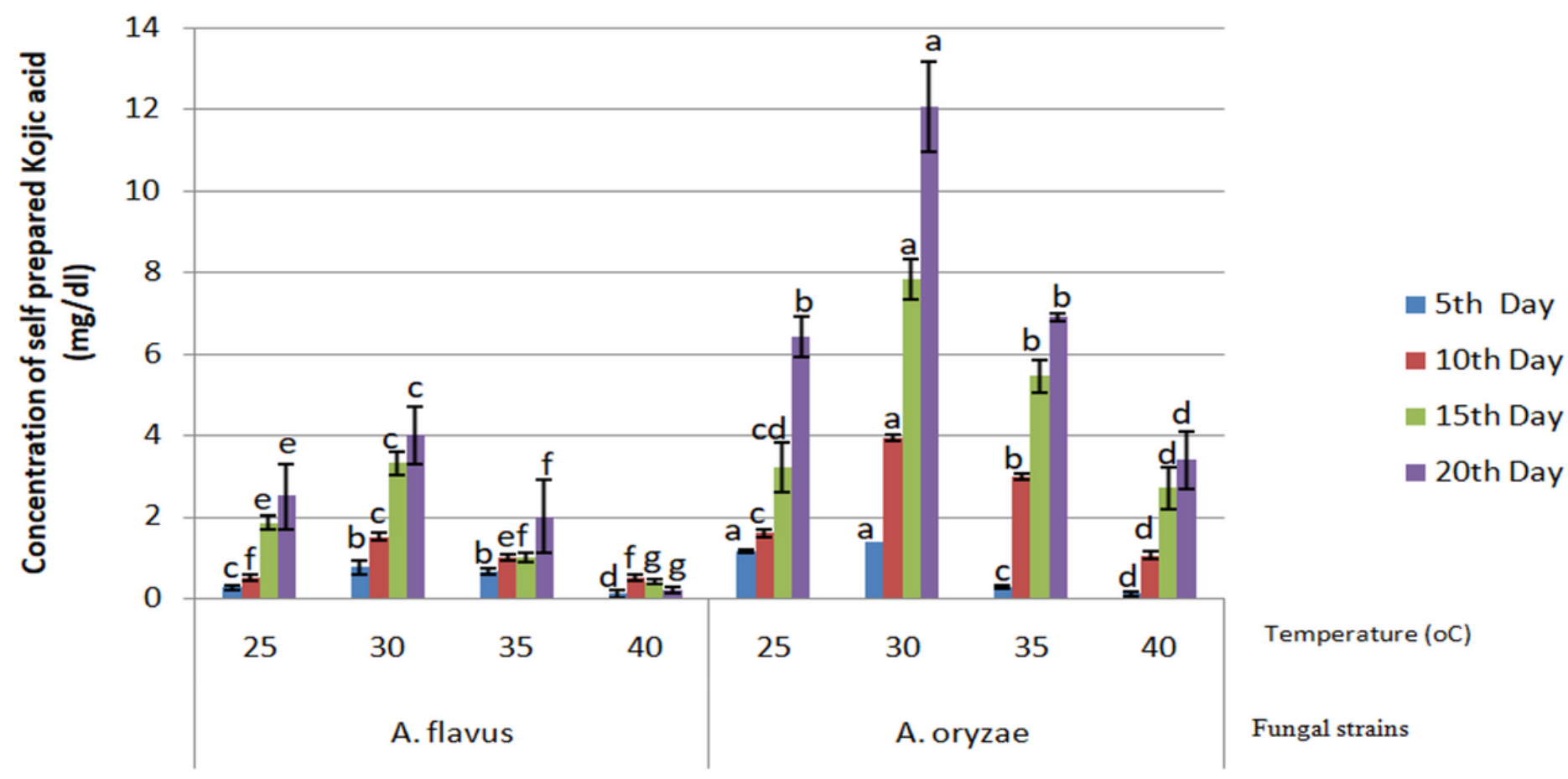

Figure 4

Concentration of self-prepared Kojic acid from A. flavus and A. oryzae at different temperature on different days. a= Maximum amount of Kojic acid (as per statistical analysis), j= Least amount of Kojic acid (as per statistical analysis) 

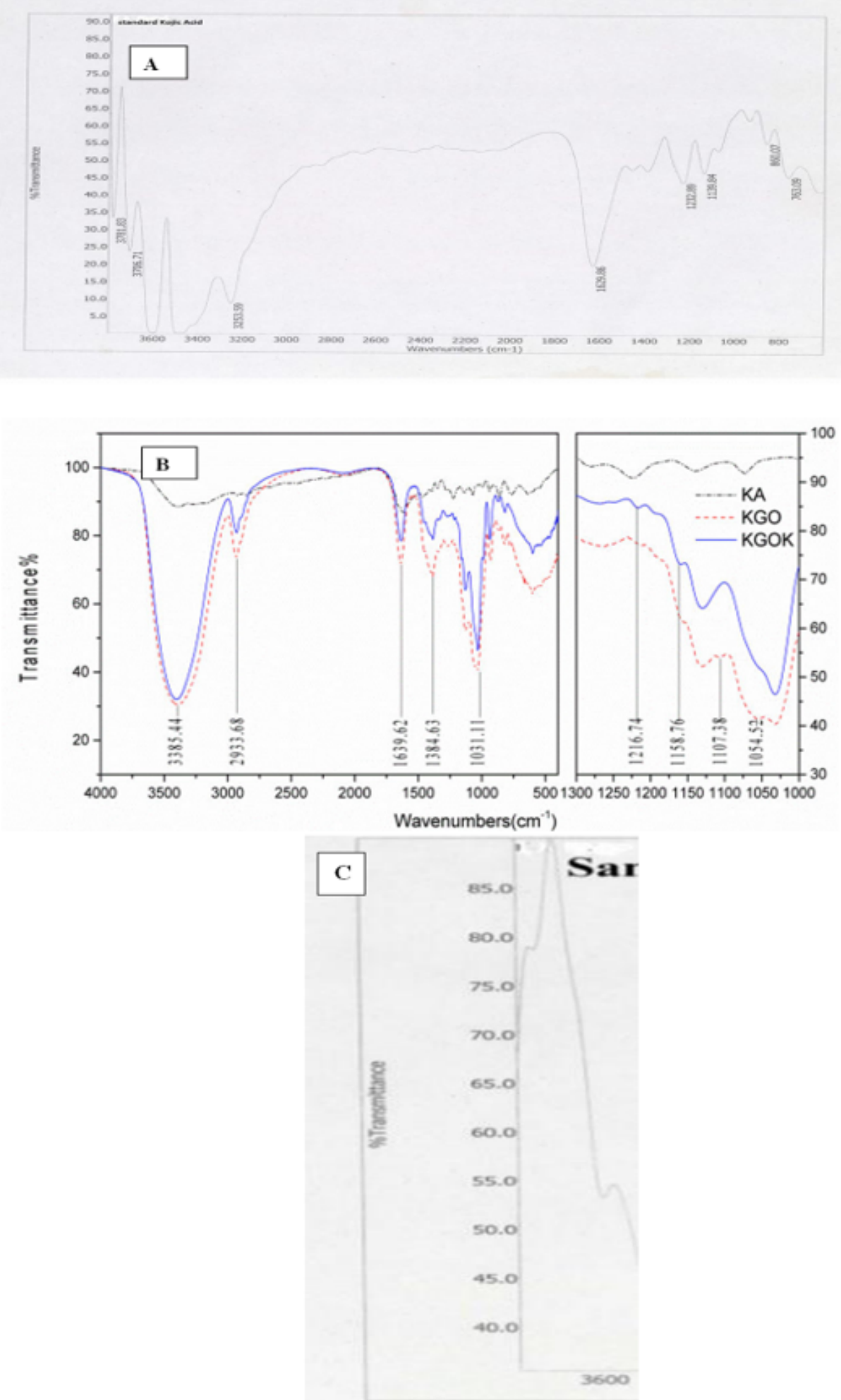

\section{Figure 5}

FTIR Spectrum of Kojic acid (KA) in the wavelength region 4000-400 $\mathrm{cm}-1$ from (A) A. flavus (B) A. oryzae (C)Standard Kojic acid crystals 

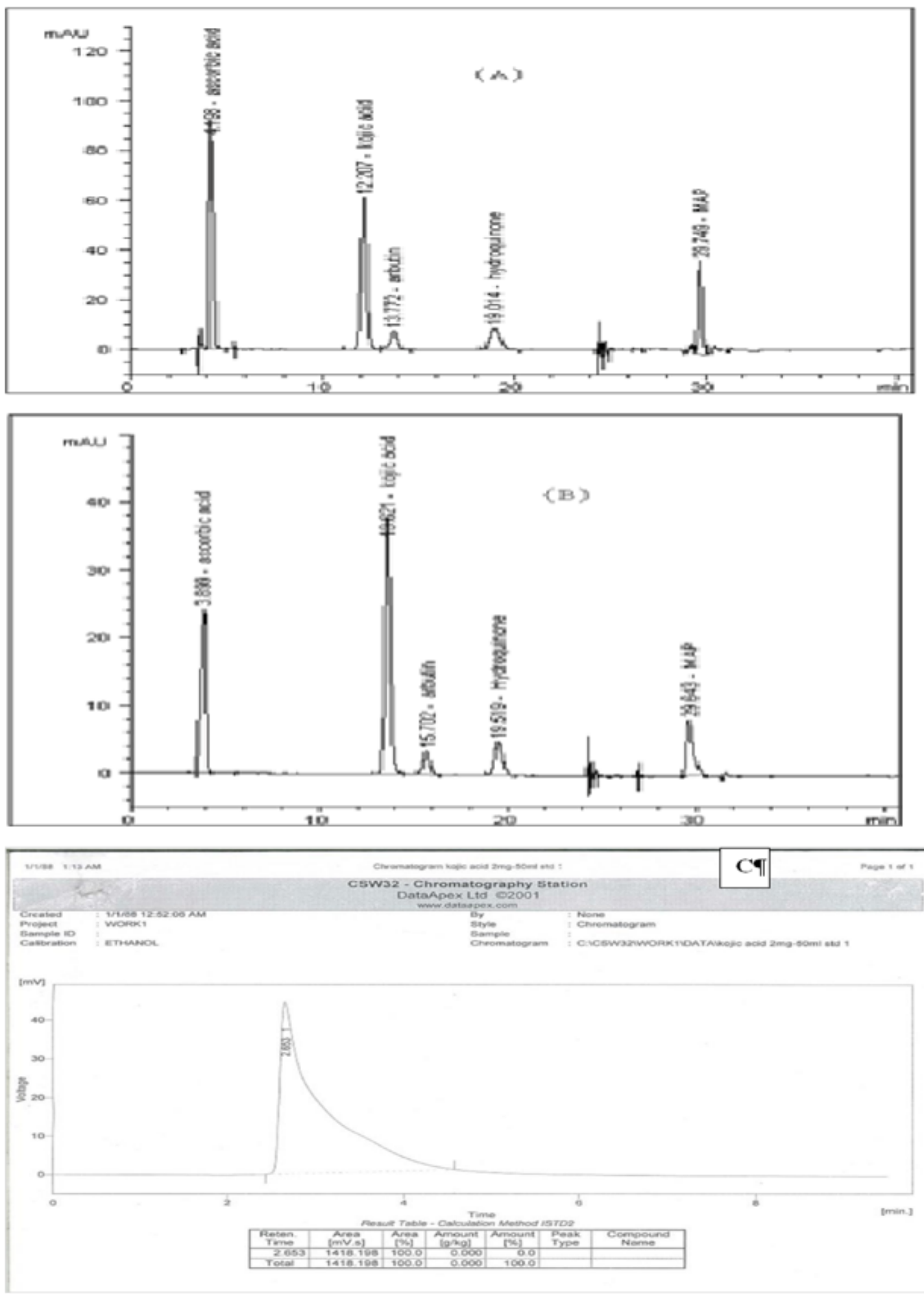

Figure 6

HPLC of standard Kojic acid shows Kojic acid peak from (A) A. flavus (B) A. oryzae (C) Standard 
Figure 7

Figure not provided in this version 
Figure 8

Figure not provided in this version 